\title{
Prolonged subjective duration near the hands: Effects of hand proximity on temporal reproduction
}

\author{
Yue $\mathrm{Qi}^{1,2} \cdot$ Xiaotao Wang ${ }^{3} \cdot$ Xiaosong $\mathrm{He}^{1,4} \cdot$ Feng $\mathrm{Du}^{1,2}$ \\ Published online: 29 May 2019 \\ (C) The Psychonomic Society, Inc. 2019
}

\begin{abstract}
It has been reported that human visual perception and attention are altered when the hands are nearby. Previous studies indicate that placing hands near stimuli enhances a subject's temporal sensitivity. However, few researchers have investigated the effect of hand proximity on reproducing temporal duration. Moreover, the delayed attentional disengagement and enhanced magnocellular visual processing theories provide two distinct predictions of the hand proximity effect on reproduced duration. Delayed attentional disengagement near hands will cause prolonged reproductions, whereas enhanced magnocellular visual processing predicts more accurate reproduction in the peri-hand space. The current study is the first to show that a short temporal duration is reproduced for a longer period near hands than far from hands in the dual-responding-hand condition, and this handproximity effect is attenuated in the single-responding-hand condition. These findings together with two further studies suggest that reproducing a temporal duration is modulated by hand proximity through prolonged attentional switch.
\end{abstract}

Keywords Hand position $\cdot$ Near-hand space $\cdot$ Time perception $\cdot$ Attention $\cdot$ Temporal reproduction

\section{Introduction}

Converging evidence has reported that human visual perception and attention are altered when the hands are nearby (Abrams, Davoli, Du, Knapp III, \& Paull, 2008; Brockmole, Davoli, Abrams, \& Witt, 2013; Davoli, Du, Montana, Garverick, \& Abrams, 2010; Wang, Du, He, \& Zhang, 2014). For example, attention shifts more slowly from item to item when the items are in the vicinity of the hands

Electronic supplementary material The online version of this article (https://doi.org/10.3758/s13423-019-01614-9) contains supplementary material, which is available to authorized users.

Feng Du

duf@psych.ac.cn

1 CAS Key Laboratory of Behavioral Science, Institute of Psychology, Chinese Academy of Sciences, 16 Lincui Road, Chaoyang District, Beijing 100101, China

2 Department of Psychology, University of Chinese Academy of Sciences, Beijing, China

3 School of Psychology, Jiangxi Normal University, Nanchang, Jiangxi, China

4 Department of Bioengineering, University of Pennsylvania, Philadelphia, PA, USA compared with when they are far away from the hands (Abrams et al., 2008; Davoli, Brockmole, Du, \& Abrams, 2012). Similarly, salient distractors presented in the perihand space relative to the far-distant space cause longer delays in observers' responses to target stimuli (Abrams et al., 2008; Vatterott \& Vecera, 2013). These findings suggest that spatial attention is more difficult to disengage from stimuli closer to hands than far from hands, and such longer attentional allocation may lead to elaborated processing of items near hands.

People's ability to estimate time can be manifested as temporal sensitivity and subjective duration (Kopec \& Brody, 2010; Wearden \& Jones, 2013). Temporal sensitivity helps people distinguish subtle differences among different durations, and reflects people's ability for temporal discrimination (Wiener, Lohoff, \& Coslett, 2011; Wittmann, 2013). Previous research has shown that placing hands near stimuli enhances a subject's temporal sensitivity (Goodhew, Gozli, Ferber, \& Pratt, 2013; Gozli, West, \& Pratt, 2012). Subjective duration is the awareness and experience of successive moments over time, which reflects a person's perception of objective duration (Wittmann, 2013; Wittmann, Simmons, Aron, \& Paulus, 2010). However, the two theoretical accounts of the hand proximity effect make distinct predictions regarding subjective duration. 
Previous research proposes that subjective temporal duration is modulated by attention (Cai, Connell, \& Holler, 2013; DeLong, 1981; Tse, Intriligator, Rivest, \& Cavanagh, 2004; Xuan, Zhang, He, \& Chen, 2007; Yates, Loetscher, \& Nicholls, 2012). For example, temporal duration is perceived to be longer when people pay more attention to the stimuli (Cai et al., 2013; Thomas \& Weaver, 1975). In contrast, temporal duration will be judged to be shorter with less engagement of attention (e.g., a secondary task) (Casini \& Macar, 1997; Noreika, Falter, \& Rubia, 2013). Since stimuli near the hands receive prolonged allocation of attention or delayed attentional disengagement compared with stimuli far from the hands (Abrams et al., 2008; Reed, Grubb, \& Steele, 2006), we expected that hand proximity should make participants overestimate temporal duration through an altered attentional process.

On the other hand, some studies suggest that objects near hands bias processing towards an action-oriented magnocellular visual pathway, resulting in a low spatial resolution but high temporal resolution (Goodhew et al., 2013; Gozli et al., 2012). This neural mechanism results in increased temporal sensitivity and decreased spatial sensitivity for stimuli near hands compared with far from hands (Gozli et al., 2012). Thus, people might have more accurate temporal reproduction when objects are near their hands than when they are far from their hands. However, few researchers have investigated the effect of hand proximity on subjective duration.

The present study employed a temporal reproduction task to explore whether subjective temporal duration would be modulated by the position of the hands. In Experiments 1 and 2, participants were asked to respond with both hands. In Experiment 3, participants were asked to respond with a single hand to reduce attentional switch while maintaining hand positions as in Experiments 1 and 2. If stimuli near hands truly bias processing to a magnocellular visual pathway, participants would better discriminate the difference between sample interval and reproduced interval, thus resulting in a more accurate temporal reproduction. In addition, they should behave consistently with dual-responding hands (Experiments 1 and 2) and a single-responding hand (Experiment 3). However, if stimuli near the hands delay attentional disengagement, participants would overestimate temporal durations with dual-responding hands. But participants would have less overestimation of temporal durations with a single-responding hand because of less attentional switch in this condition.

\section{Experiment 1}

This experiment was designed to test whether hand proximity can modulate temporal reproduction when sample intervals were $1,200 \mathrm{~ms}, 1,600 \mathrm{~ms}$, and 2,000 ms.

\section{Methods}

\section{Participants}

This study was approved by the internal review board of the Institute of Psychology, Chinese Academy of Sciences. In Experiments 1 and 2, given there had been no similar experiment conducted before, we conducted an a priori power analysis with an estimate of $\eta_{p}^{2}=0.30$, which was consistent with previous results (see Thomas \& Sunny, 2017; Wang, Du, He, $\&$ Zhang, 2014). Twenty-two participants were needed to detect an effect of hand condition with a power of $0.80(\alpha=$ 0.05 ). Twenty-eight students were paid to participate in this experiment. They all provided signed informed consent before participation. All of them had normal or corrected-to-normal vision. Participants were naïve with regard to the purpose of the experiment.

\section{Apparatus, stimuli, and procedure}

The experiment closely followed the method of the Abrams et al. (2008) study. Stimuli were presented on a 17-in. CRT monitor at a viewing distance of $43 \mathrm{~cm}$. Participants steadied their head by resting on a chinrest. In the hand-proximal condition, subjects rested their elbows on cushions on the table and held each hand on a mouse that was attached to the edges of the monitor aligned with the middle of the CRT. In the hand-distal condition, subjects supported a lightweight 38$\mathrm{cm}$ long board on their laps upon which was mounted two mice identical to those in the hand-proximal condition. Subjects held one hand on each mouse.

All stimuli were presented on a black background. The sequence of events of each trial is illustrated in Fig. 1. Each trial began with a warning sign stating "Attention please" at the center of the screen for 1,000 ms. This was followed by a central fixation cross for a random interval from $800 \mathrm{~ms}$ to $1,000 \mathrm{~ms}$, and then a blank screen for a random interval varying from $400 \mathrm{~ms}$ to $600 \mathrm{~ms}$. A gray square with a length of $12.5^{\circ}$ for each side was presented as a sample stimulus at the center of the screen. The sample stimulus lasted 1,200 ms, $1,600 \mathrm{~ms}$, or 2,000 ms for equal probability. After the sample stimulus disappeared, another instruction on the screen stating "press left button to start and right button to end time reproduction" was presented until the left button was pressed. Participants were told to remember how long the sample stimulus (the first gray square) lasted, and then press the right button of the left mouse to start and the left button of the right mouse to stop their temporal reproduction. Once the left button was pressed, the second gray square, which was physically the same as the first gray square, was presented as a response stimulus. Participants had to press the right button to terminate the presentation of the response stimulus when they thought it had lasted for the same amount of time as the sample stimulus. 

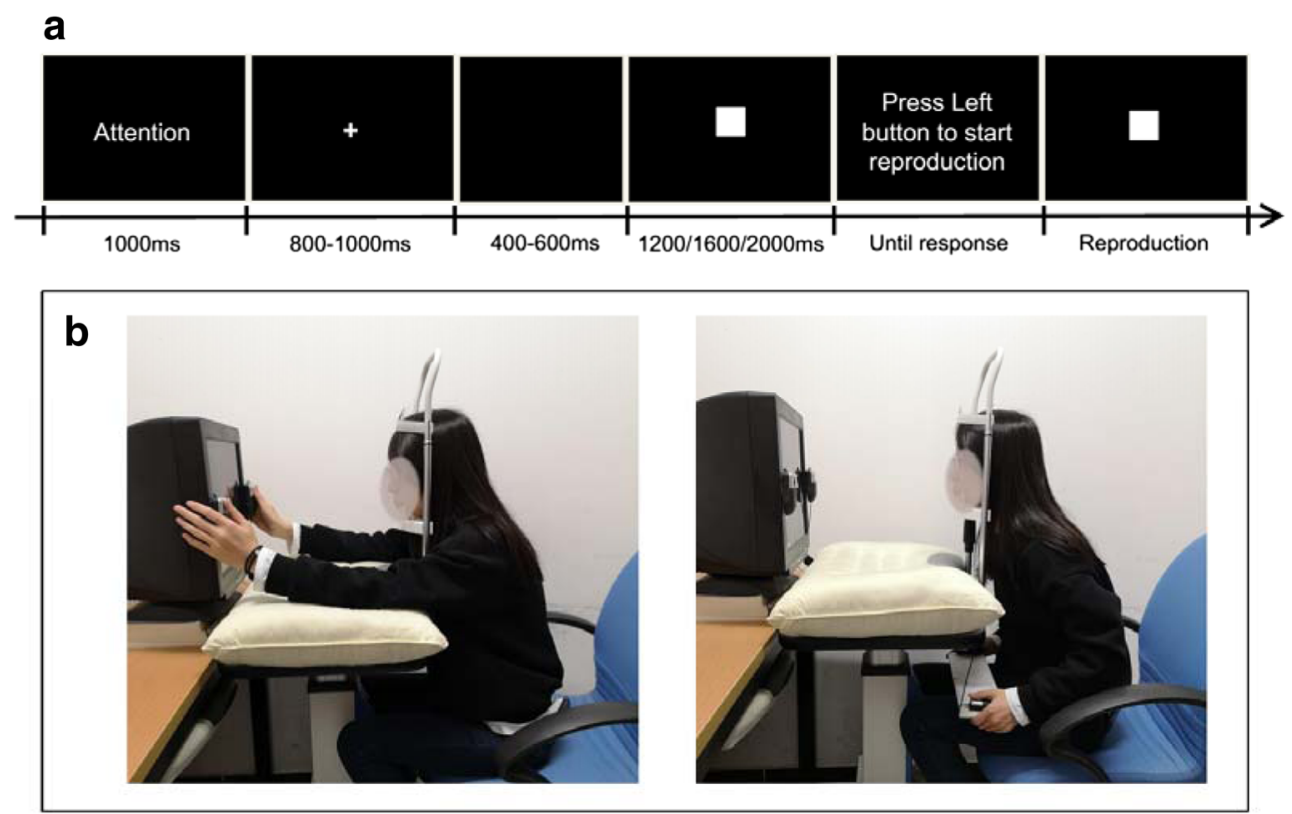

Fig. 1 (a) Illustration of event sequence in Experiment 1. (b) Illustration of the hand-proximal (left) and hand-distal (right) conditions in the present study

At the beginning of the experiment, each participant was asked to remove his or her watch and was told not to count during the temporal reproduction task.

\section{Design}

There were two within-subject variables: the sample interval and the hand proximity condition. First, each trial was in one of three sample intervals: $1,200 \mathrm{~ms}, 1,600 \mathrm{~ms}$, and 2,000 ms. In addition, each trial was in one of two hand-proximity conditions: hand-proximal and hand-distal conditions. There were two blocks for each of two hand-proximity conditions, altogether yielding four blocks in the experiment. The order of blocks was counterbalanced across participants. In each block, there were ten replications for each of the three sample intervals $(1,200 \mathrm{~ms}, 1,600 \mathrm{~ms}$, and 2,000 ms) in a random order, yielding 30 trials for each block and a total of 120 trials. Participants first served in one block of 15 practice trials in which the sample stimulus lasted $1,000 \mathrm{~ms}, 1,500 \mathrm{~ms}$, and $1,800 \mathrm{~ms}$. They were asked to practice under either a handproximal or a hand-distal condition to familiarize them with the first experimental block. They then completed four blocks of test trials.

\section{Results}

The ratio between the reproduced interval and the sample interval is illustrated in Fig. 2 as a function of two handproximity conditions and three sample intervals. The ratio of relative temporal reproductions exceeding one indicated that participants reproduced an interval longer than the sample intervals. A repeated-measures ANOVA showed a main effect of sample interval, $\mathrm{F}(1.28,34.47)=22.65, p<.001, \eta_{p}{ }^{2}=$ 0.456 , with the temporal reproduction ratios decreasing as the sample interval increased (Greenhouse-Geisser correction was used when sphericity was violated). The main effect of hand-proximity conditions was also significant, $\mathrm{F}(1,27)=$ $7.84, p=.009, \eta_{p}{ }^{2}=0.225$, with a higher temporal reproduction ratio for the hand-proximal condition than the hand-distal condition. The interaction between hand condition and sample interval was significant, $\mathrm{F}(2,54)=3.72, p=.031, \eta_{p}{ }^{2}=0.121$. A post hoc test (Bonferroni correction was used in all the post hoc tests throughout the paper) revealed that when the sample interval was $1,200 \mathrm{~ms}$, participants reproduced the handproximal condition for a significantly longer duration than

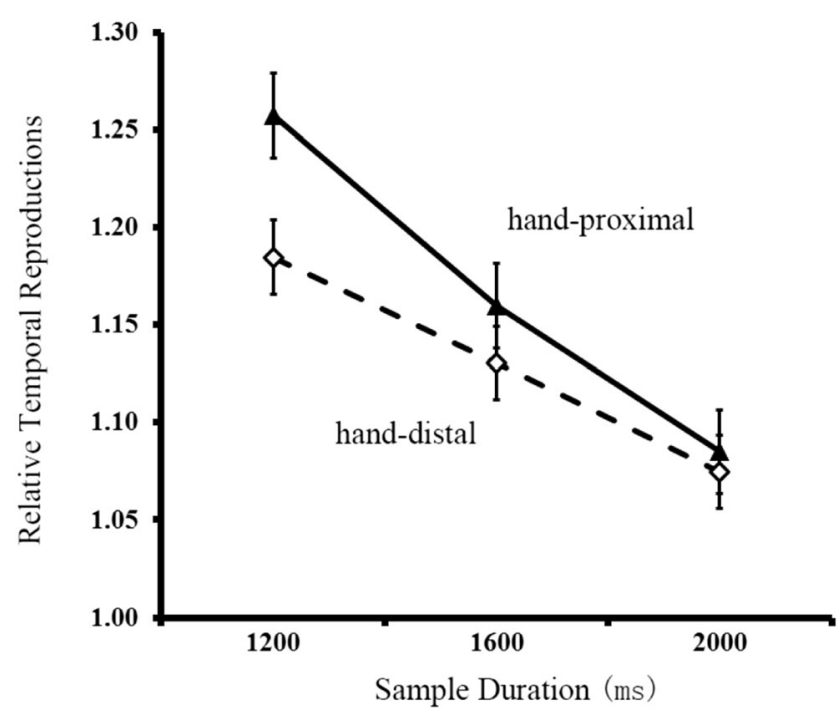

Fig. 2 Mean ratios between reproduced and sample durations in Experiment 1. Error bars represent the within-subject standard errors 
the hand-distal condition (mean difference $=0.073$, S.E. $=$ $0.024, p=.006,95 \% \mathrm{CI}=[.02, .12])$. However, there was no significant difference between two hand-proximity conditions when the sample interval was $1,600 \mathrm{~ms}$ (mean difference $=0.029$, S.E. $=0.018, p=.121,95 \%$ CI $=[-.01, .07])$ or $2,000 \mathrm{~ms}$ (mean difference $=0.011, \mathrm{~S} . \mathrm{E} .=0.013, p=.427$, $95 \% \mathrm{CI}=[-.02, .04])$.

\section{Experiment 2}

Experiment 1 found that sample intervals near hands resulted in a longer estimation of a short temporal duration $(1,200 \mathrm{~ms})$ than those far from the hands. But hand proximity did not alter temporal reproduction at longer intervals $(1,600 \mathrm{~ms}$ or 2,000 $\mathrm{ms})$. Previous studies have shown that participants tend to overestimate a sample interval shorter than 2,000 ms and gradually begin to underestimate a temporal interval when it becomes increasingly longer than 2,000 ms (Morillon, Kell, \& Giraud, 2009; Pöppel, 1997; Ulbrich, Churan, Fink, \& Wittmann, 2007; Wittmann et al., 2007; Wittmann, 2013). The hand-proximity effect may impact temporal perception in two different ways: enhanced magnocellular visual processing in the peri-hand space would lead to more accurate reproductions regardless of sample intervals, while delayed attentional disengagement may lead to longer reproduction at short intervals near hands but not at the long intervals. Thus, the present experiment was designed to test whether hand proximity had a different effect on temporal reproductions for intervals longer than 2,000 ms compared with short intervals.

\section{Methods}

Experiment 2 was identical to Experiment 1 with one exception. The target square was presented at any of three durations for $1,200 \mathrm{~ms}, 2,000 \mathrm{~ms}$, or $2,800 \mathrm{~ms}$. A further 26 students provided signed informed consent before they participated in this experiment. All of them had normal or corrected-tonormal vision. Participants were naïve with regard to the purpose of the experiment.

\section{Results}

The ratio between the reproduced interval and the sample interval is illustrated in Fig. 3. A repeated-measures ANOVA showed a main effect of sample interval, $\mathrm{F}(1.16$, $29.03)=10.28, p=.002, \eta_{p}{ }^{2}=0.291$, with decreasing temporal reproduction ratios as the sample interval increased. The main effect of hand proximity condition was not significant, $\mathrm{F}(1,25)=3.01, p=.095, \eta_{p}{ }^{2}=0.107$. Importantly, the interaction between hand condition and sample interval was significant, $\mathrm{F}(2,50)=4.55, p=.015, \eta_{p}{ }^{2}=0.154$. A post hoc test revealed that when the sample interval was $1,200 \mathrm{~ms}$

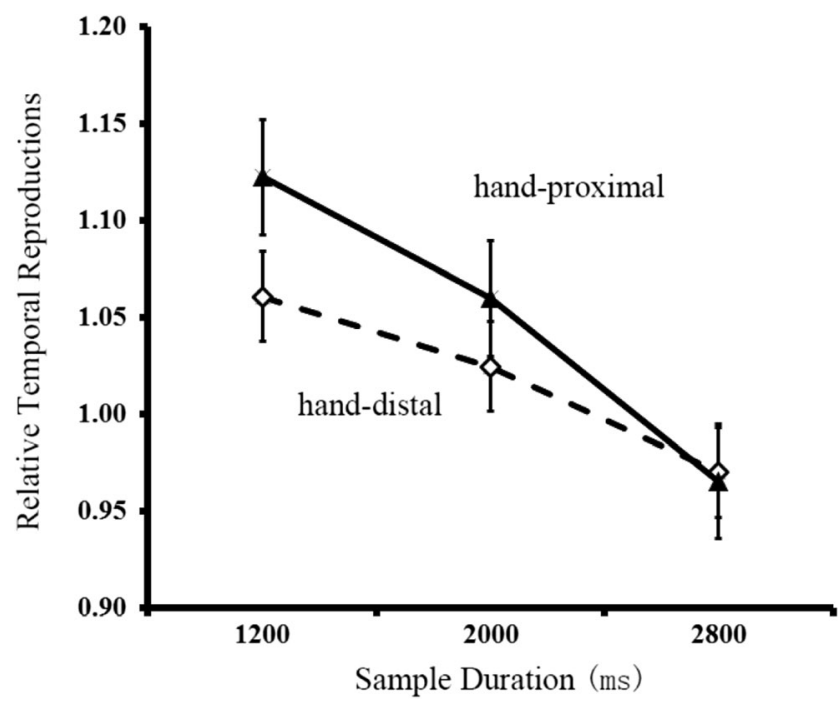

Fig. 3 Mean ratios between reproduced and sample durations in Experiment 2. Error bars represent the within-subject standard errors

participants reproduced a significantly longer duration under the hand-proximal condition than under the hand-distal condition (mean difference $=0.062$, S.E. $=0.024, p=.018,95 \%$ $\mathrm{CI}=[.01, .11])$. However, there was no significant difference between two hand-proximity conditions when the sample interval was $2,000 \mathrm{~ms}$ (mean difference $=0.035$, S.E. $=0.023, p$ $=.132,95 \% \mathrm{CI}=[-.01, .08]$ ) or $2,800 \mathrm{~ms}$ (mean difference $=$ 0.005 , S.E. $=0.018, p=.798,95 \% \mathrm{CI}=[-.04, .03])$. Moreover, consistent with previous studies (Ulbrich et al., 2007; Wittmann et al., 2007), participants tended to overestimate sample intervals at short intervals $(1,200$ and 2,000 ms) but underestimate at longer intervals $(2,800 \mathrm{~ms})$.

Experiment 2 further confirmed our previous findings in Experiment 1, and showed that people tended to overshoot more in reproducing a short sample interval $(1,200 \mathrm{~ms})$ when the stimulus was near hands compared with when far from hands. However, this hand-proximity effect was absent at sample intervals of $2,000 \mathrm{~ms}$ or $2,800 \mathrm{~ms}$.

\section{Experiment 3}

Experiments 1 and 2 showed that short intervals near the hands were overestimated more than those far from the hands when participants were required to respond with both hands. But long intervals near the hands were reproduced for as long as those far from the hands. Attentional switch costs between two responding hands increase as the interval decreases (Gálvez-García, Gabaude, De la Rosa, \& Gomez, 2014; Gálvez-García, Peña, Albayay, \& Cohen, 2018; Garner, Tombu, \& Dux, 2014), thus attentional switch between two responding hands influences temporal reproduction for a short interval more than for a long interval. In addition, previous studies showed differences between one-hand and two-hand 
configurations (Bush \& Vecera, 2014). It was unknown whether temporal reproduction would change when Experiment 3 required participants to respond with a single hand while maintaining two same-hand positions as in Experiments 1 and 2. We expected a smaller effect of hand proximity because of reduced attentional switch between two responding hands in Experiment 3.

\section{Methods}

Experiment 3 was identical to Experiment 2 with two exceptions. First, to distinguish the sample and response stimulus more easily, the target stimulus was changed into a circle with the same color, brightness, and proportion as the square. Second, participants were told to always press a single button during the experiment. We estimated an optimal sample size by using $G^{*}$ Power for $\alpha=0.05$ and power $=0.80$ (Saccone et al., 2018). Since the hand-proximity effect in the current experiment was expected to be slightly attenuated compared with Experiments 1 and 2, 35-74 participants were required to detect a small effect of hand condition if $\eta 2_{p}$ varied from 0.10 to 0.20 . Thus another 48 naïve students were randomly assigned to the two groups and asked to press either the left or the right button during the experiment.

\section{Results}

The ratio between the reproduced interval and the sample interval is illustrated in Fig. 4. A repeated-measures ANOVA showed a main effect of sample interval, $\mathrm{F}(1.15$, $53.81)=29.83, p<.001, \eta_{p}{ }^{2}=0.388$, with the temporal reproduction ratios decreasing as sample interval increased. The main effect of hand condition was also significant, $\mathrm{F}(1$,

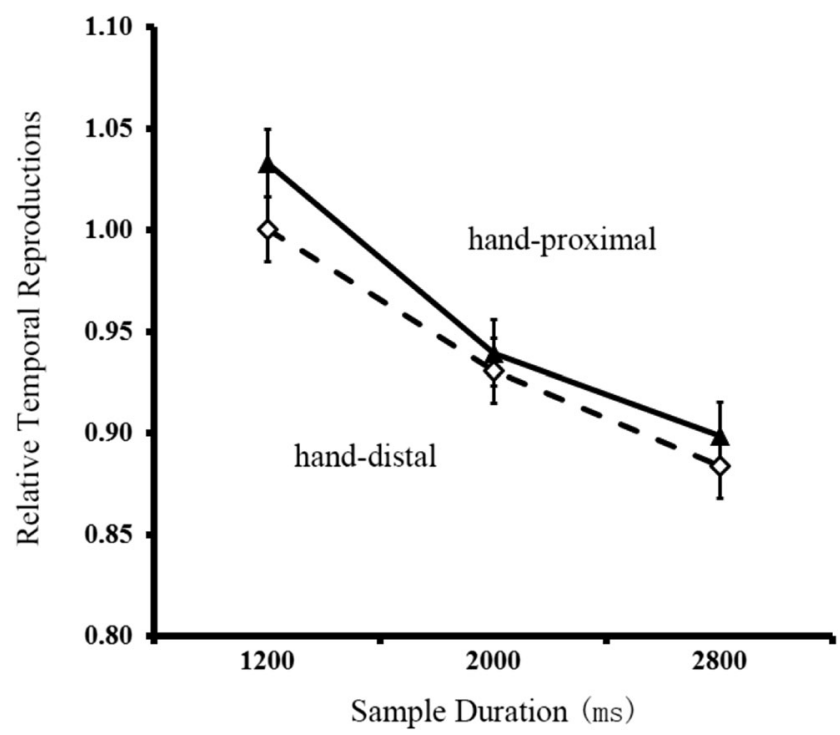

Fig. 4 Mean ratios between reproduced and sample durations in Experiment 3. Error bars represent the within-subject standard errors
$47)=4.13, p=.048, \eta_{p}^{2}=0.081$, with higher temporal reproduction ratios for the hand-proximal condition than the handdistal condition. The interaction between hand condition and sample interval was not significant, $\mathrm{F}(2,94)=1.17, p=.315$, $\left.\eta_{p}{ }^{2}=0.024\right)$.

In addition, we analyzed the differences between the dualand single-hand response conditions, by conducting 2 hand proximity conditions $\times 3$ sample intervals $(1,200 \mathrm{~ms}, 2,000$ $\mathrm{ms}$, and 2,800 ms) $\times 2$ tasks (Experiment 2 and Experiment 3 ) ANOVA on temporal reproduction ratios. The results showed significant main effects of hand proximity $(\mathrm{F}(1,72)=7.52, p$ $\left.=.008, \eta_{p}{ }^{2}=0.095\right)$ and sample interval $(\mathrm{F}(1.15,83.04)=$ $\left.34.19, p<.001, \eta_{p}{ }^{2}=0.322\right)$. The interaction between hand condition and sample interval was also significant, $\mathrm{F}(2,144)$ $=4.83, p=.009, \eta_{p}{ }^{2}=0.063$. A post hoc test revealed that when the sample interval was $1,200 \mathrm{~ms}$, participants reproduced a significantly longer duration under the handproximal condition than under the hand-distal condition, mean difference $=0.047$, S.E. $=0.014, p=.001,95 \% \mathrm{CI}=[.02, .08]$. When the sample interval was $2,000 \mathrm{~ms}$ (mean difference $=$ 0.022 , S.E. $=0.012, p=.076,95 \% \mathrm{CI}=[-.002, .05])$ or $2,800 \mathrm{~ms}$ (mean difference $=0.005$, S.E. $=0.009, p=.596$, $95 \% \mathrm{CI}=[-.01, .02])$, there was no difference between the two hand conditions. The main effect of task was significant, $\mathrm{F}$ (1, 72) $=7.01, p=.010, \eta_{p}{ }^{2}=0.089$, and the temporal reproduction ratios were decreasing under the single-hand condition. The other interactions were not significant (all $p \mathrm{~s}>.05$ ). These results indicated that although the effect of hand proximity was attenuated, participants still tended to overestimate short intervals $(1,200 \mathrm{~ms})$ when stimuli were near their hands.

\section{General discussion}

Experiments 1 and 2 consistently showed participants made greater overestimations of a short temporal interval $(1,200 \mathrm{~ms})$ for the hands-proximal condition than for the hands-distal condition when both hands are required to respond. However, such an overestimation effect of hand proximity reduced as intervals increased $(1,600 \mathrm{~ms}, 2,000 \mathrm{~ms}$, and $2,800 \mathrm{~ms}$ ). This is the first study to show that hand proximity modulates temporal reproduction. Furthermore, Experiment 3 showed this effect was attenuated in the single-hand response condition. These findings indicate the effect of hand proximity on the subjective duration might depend on attentional switch.

Our results are consistent with some previous studies that suggested that attentional switch is delayed with hands nearby (Abrams et al., 2008; Thomas \& Sunny, 2017). By employing the additional singleton paradigm, Thomas and Sunny (2017) found that either a distractor or the target displayed near the hands slowed down responses more than far from the hands. Previous research also showed that volitional switches among different attentional scopes were delayed near the hands 
(Davoli et al., 2012). In our current studies, participants had to respond with two hands in Experiments 1 and 2, and respond with a single hand in Experiment 3. Compared with the singlehand condition, participants needed to make more attentional switches in the dual-hand condition. Thus, additional switch costs in the dual-hand condition increased the effect of hand proximity on temporal reproduction compared with the singlehand condition. Further studies are encouraged to test whether hand proximity delays attentional switch between non-visual stimuli.

Prolonged attentional switch costs between two hands instead of altered time perception might account for the effect of hand proximity on temporal reproduction in the present study. Since there is no attentional switch between hands in verbal estimation or temporal bisection task, hand proximity should not affect temporal estimation in these tasks. As expected, in both verbal estimation and the temporal bisection task, hand proximity has no effect on time perception (see Supplemental Materials for detailed results), which was consistent with Aday et al.'s study (under review). Taking these studies altogether, hand proximity has no effect on time perception when attentional switch between hands is eliminated (e.g., the single-responding-hand condition in Experiment 3, verbal estimation or temporal bisection task in the Supplemental Material and Aday et al.'s study).

Moreover, the effect of hand proximity on subjective duration is only found when the sample interval is short. These findings are consistent with previous studies on the psychological refractory period (PRP) effect that showed that people respond more slowly as the interval between two successive stimuli is decreased (Garner, Tombu, \& Dux, 2014). Thus, delayed attentional switch has a greater impact at short intervals compared with long intervals. Therefore, we can easily find an effect of hand proximity on the reproductions for short intervals, but not for long durations.

Our results cannot be fully explained by enhanced processing on the magnocellular visual pathway with proximal hands (Goodhew, Edwards, Ferber, \& Pratt, 2015; Goodhew et al., 2013; Gozli et al., 2012). Enhanced magnocellular processing provides more sensitive temporal acuity near hands (Goodhew et al., 2013; Gozli et al., 2012), possibly because the magnocellular visual pathway is more sensitive to rapid changes in luminance (Chase \& Jenner, 1993; Merigan, Byrne, \& Maunsell, 1991). Hence, if the sample duration was short enough (e.g., less than $100 \mathrm{~ms}$ ), the temporal perception might be altered near hands. But when the duration was longer than 1,200 $\mathrm{ms}$ in our experiments, the handproximity effect on temporal perception might be negligible. Further research is encouraged to explore whether the handproximity effect affects the temporal perception of short durations.

In summary, the current research showed that a relatively short temporal duration is overestimated more near hands than far from hands through prolonged attentional switch. These findings also have implications for the design of touch-screen applications because prolonged perception of temporal duration degrades the user experience.

Acknowledgements We thank Bin Zhou for his valuable suggestions for the manuscript. This research was supported by the National Natural Science Foundation of China (31470982); the Scientific Foundation of the Institute of Psychology, Chinese Academy of Sciences (Y4CX033008, Y5CX122005).

\section{References}

Abrams, R. A., Davoli, C. C., Du, F., Knapp III, W. H., \& Paull, D. (2008). Altered vision near the hands. Cognition, 107(3), 10351047.

Brockmole, J. R., Davoli, C. C., Abrams, R. A., \& Witt, J. K. (2013). The world within reach: Effects of hand posture and tool use on visual cognition. Current Directions in Psychological Science, 22(1), 3844.

Cai, Z. G., Connell, L., \& Holler, J. (2013). Time does not flow without language: Spatial distance affects temporal duration regardless of movement or direction. Psychonomic Bulletin \& Review, 20(5), 973-980.

Casini, L., \& Macar, F. (1997). Effects of attention manipulation on judgments of duration and of intensity in the visual modality. Memory \& Cognition, 25(6), 812-818.

Chase, C., \& Jenner, A. R. (1993). Magnocellular visual deficits affect temporal processing of dyslexics. Annals of the New York Academy of Sciences, 682(1), 326-329.

Davoli, C. C., Brockmole, J. R., Du, F., \& Abrams, R. A. (2012). Switching between global and local scopes of attention is resisted near the hands. Visual Cognition, 20(6), 659-668.

Davoli, C. C., Du, F., Montana, J., Garverick, S., \& Abrams, R. A. (2010). When meaning matters, look but don't touch: The effects of posture on reading. Memory \& Cognition, 38(5), 555-562.

DeLong, A. (1981). Phenomenological space-time: Toward an experiential relativity. Science, 213(4508), 681-683.

Gálvez-García, G., Gabaude, C., De la Rosa, F. D., \& Gomez, E. (2014). Influence of prior use of the same or different effectors in a reaching action. Perceptual and Motor Skills, 118(1), 126-144.

Gálvez-García, G., Peña, J., Albayay, J., \& Cohen, H. (2018). Hand switching costs are not uniform across response components. Journal of Motor Behavior, 1-6.

Garner, K. G., Tombu, M. N., \& Dux, P. E. (2014). The influence of training on the attentional blink and psychological refractory period. Attention, Perception, \& Psychophysics, 76(4), 979-999.

Goodhew, S. C., Edwards, M., Ferber, S., \& Pratt, J. (2015). Altered visual perception near the hands: A critical review of attentional and neurophysiological models. Neuroscience \& Biobehavioral Reviews, 55, 223-233.

Goodhew, S. C., Gozli, D. G., Ferber, S., \& Pratt, J. (2013). Reduced temporal fusion in near-hand space. Psychological Science, 24(6), 891-900.

Gozli, D. G., West, G. L., \& Pratt, J. (2012). Hand position alters vision by biasing processing through different visual pathways. Cognition, 124(2), 244-250.

Kopec, C. D., \& Brody, C. D. (2010). Human performance on the temporal bisection task. Brain and Cognition, 74(3), 262-272. 
Merigan, W. H., Byrne, C. E., \& Maunsell, J. H. (1991). Does primate motion perception depend on the magnocellular pathway? Journal of Neuroscience, 11(11), 3422-3429.

Morillon, B., Kell, C. A., \& Giraud, A.-L. (2009). Three stages and four neural systems in time estimation. The Journal of Neuroscience, 29(47), 14803-14811.

Noreika, V., Falter, C. M., \& Rubia, K. (2013). Timing deficits in attention-deficit/hyperactivity disorder (ADHD): Evidence from neurocognitive and neuroimaging studies. Neuropsychologia, 51(2), 235-266.

Pöppel, E. (1997). A hierarchical model of temporal perception. Trends in Cognitive Sciences, 1(2), 56-61.

Reed, C. L., Grubb, J. D., \& Steele, C. (2006). Hands up: Attentional prioritization of space near the hand. Journal of Experimental Psychology: Human Perception and Performance, 32(1), 166-177.

Saccone, E. J., Szpak, A., Churches, O., \& Nicholls, M. E. (2018). Close interpersonal proximity modulates visuomotor processing of object affordances in shared, social space. Attention, Perception, \& Psychophysics, 80(1), 54-68.

Thomas, E. A. C., \& Weaver, W. B. (1975). Cognitive processing and time perception. Perception \& Psychophysics, 17(4), 363-367.

Thomas, T., \& Sunny, M. M. (2017). Slower attentional disengagement but faster perceptual processing near the hand. Acta Psychologica, 174, 40-47.

Tse, P. U., Intriligator, J., Rivest, J., \& Cavanagh, P. (2004). Attention and the subjective expansion of time. Perception \& Psychophysics, 66(7), 1171-1189.

Ulbrich, P., Churan, J., Fink, M., \& Wittmann, M. (2007). Temporal reproduction: Further evidence for two processes. Acta Psychologica, 125(1), 51-65.

Vatterott, D. B., \& Vecera, S. P. (2013). Prolonged disengagement from distractors near the hands. Frontiers in Psychology, 4, 533.

Wang, X., Du, F., He, X., \& Zhang, K. (2014). Enhanced spatial stimulusresponse mapping near the hands: The Simon effect is modulated by hand-stimulus proximity. Journal of Experimental Psychology: Human Perception and Performance, 40(6), 2252-2265.

Wearden, J. H., \& Jones, L. A. (2013). Explaining between-group differences in performance on timing tasks. Quarterly Journal of Experimental Psychology, 66(1), 179-199.

Wiener, M., Lohoff, F. W., \& Coslett, H. B. (2011). Double dissociation of dopamine genes and timing in humans. Journal of Cognitive Neuroscience, 23(10), 2811-2821.

Wittmann, M. (2013). The inner sense of time: How the brain creates a representation of duration. Nature Reviews Neuroscience, 14, 217.

Wittmann, M., Carter, O., Hasler, F., Cahn, B. R., Grimberg, U., Spring, P., ... Vollenweider, F. X. (2007). Effects of psilocybin on time perception and temporal control of behaviour in humans. Journal of Psychopharmacology, 21(1), 50-64.

Wittmann, M., Simmons, A. N., Aron, J. L., \& Paulus, M. P. (2010). Accumulation of neural activity in the posterior insula encodes the passage of time. Neuropsychologia, 48(10), 3110-3120.

Xuan, B., Zhang, D., He, S., \& Chen, X. (2007). Larger stimuli are judged to last longer. Journal of Vision, 7(10), 2.

Yates, M. J., Loetscher, T., \& Nicholls, M. E. R. (2012). A generalized magnitude system for space, time, and quantity? A cautionary note. Journal of Vision, 12(7), 9.

\section{Open Practices Statement}

None of the data or materials for the experiments reported here are available, and none of the experiments were preregistered.

Publisher's note Springer Nature remains neutral with regard to jurisdictional claims in published maps and institutional affiliations. 